\title{
Sub-barrier pathways to Freeman resonances
}

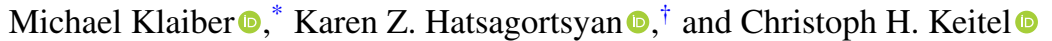 \\ Max-Planck-Institut für Kernphysik, Saupfercheckweg 1, 69117 Heidelberg, Germany
}

(Received 8 June 2020; accepted 22 October 2020; published 5 November 2020)

\begin{abstract}
The problem of Freeman resonances [Freeman et al., Phys. Rev. Lett. 59, 1092 (1987)] when strong-field ionization is enhanced due to transient population of excited Rydberg states during ionization is revisited. An intuitive model is put forward which explains the mechanism of intermediate population of excited states during nonadiabatic tunneling ionization via under-the-barrier recollision and recombination. The theoretical model is based on perturbative strong-field approximation (SFA), where the sub-barrier bound-continuum-bound pathway is described in the second-order SFA, with further ionization from the excited state by an additional perturbative step. The enhancement of ionization is shown to arise due to constructive interference of contributions into the excitation amplitudes originating from different laser cycles. The applied model provides an intuitive understanding of the electron dynamics during a Freeman resonance in strong-field ionization, as well as a means of enhancing the process and possible applications to related processes.
\end{abstract}

DOI: 10.1103/PhysRevA.102.053105

\section{INTRODUCTION}

The enhancement of strong-field ionization due to transient excitation of Stark-shifted bound states is well known from experiments in the multiphoton regime of ionization and is termed as Freeman resonances [1-13]. It is assumed that the excitation at Freeman resonances happens due to a bound-bound multiphoton transition, when the electron wave function during transition is localized within the binding potential. With increased laser intensity, the tunneling through the laser suppressed Coulomb barrier becomes dominant and the bound electron moves from the ground state immediately to the continuum. Strong-field approximation (SFA) [14-16] describes successfully direct strong-field ionization in tunneling and multiphoton regimes as well as in the intermediate nonadiabatic regime $[17,18]$, when the electron gains energy during the tunneling [19]. The quantum orbit picture $[20,21]$ which stems from the SFA description, applying saddle-point approximation (SPA) in calculation of integrals in S-matrix amplitude, provides intuitive understanding of strong-field ionization processes. Can the quantum orbit picture be extended to interpret the electron dynamics at Freeman resonances?

In the tunneling regime the atom excitation due to boundbound transitions is not probable, because it is overwhelmed by electron tunneling into the continuum. In nonadiabatic

\footnotetext{
*klaiber@mpi-hd.mpg.de

${ }^{\dagger}$ k.hatsagortsyan@mpi-hd.mpg.de
}

Published by the American Physical Society under the terms of the Creative Commons Attribution 4.0 International license. Further distribution of this work must maintain attribution to the author(s) and the published article's title, journal citation, and DOI. Open access publication funded by the Max Planck Society. tunneling, excitations can happen only when the electron revisits the atomic core, i.e., at recollisions. However, common recollisions via excursion in the real continuum [22] are accompanied by a large spreading of the electron wave packet, which reduces significantly the recollision probability. Recently, it has been recognized that recollision can happen also within the sub-barrier dynamics during tunneling [23]. The latter may contribute to the electron transition to the excited state as long as the electron gains sufficient energy during the nonadiabatic tunneling.

In this paper we develop a theory for Freeman resonances in the nonadiabatic tunneling regime which is based on the concept of under-the-barrier recollision. We employ SFA, treating the recollision with the atomic core within a perturbative approach. The resonant channel of ionization is described within the next-order perturbation of SFA. The given description allows for an interpretation of the process as taking place via sub-barrier recollision with increasing energy in the nonadiabatic regime and transition to the excited state, with further ionization after some time delay (see the interaction scheme in Fig. 1). The proposed model provides a physical explanation via the quantum orbit picture for the resonantly enhanced strong-field ionization involving excited states at a Freeman resonance.

The paper is organized as follows. The theoretical model is described in Sec. II. The half-cycle and multicycle contributions to the Freeman resonances are discussed in Secs. III A and III B, respectively. The photoelectron spectra within the present model are presented in Sec. III C, and our conclusion is given in Sec. IV.

\section{THEORETICAL MODEL}

Our main aim is to provide an intuitive picture within the scheme for Freeman resonances. For this purpose it is 


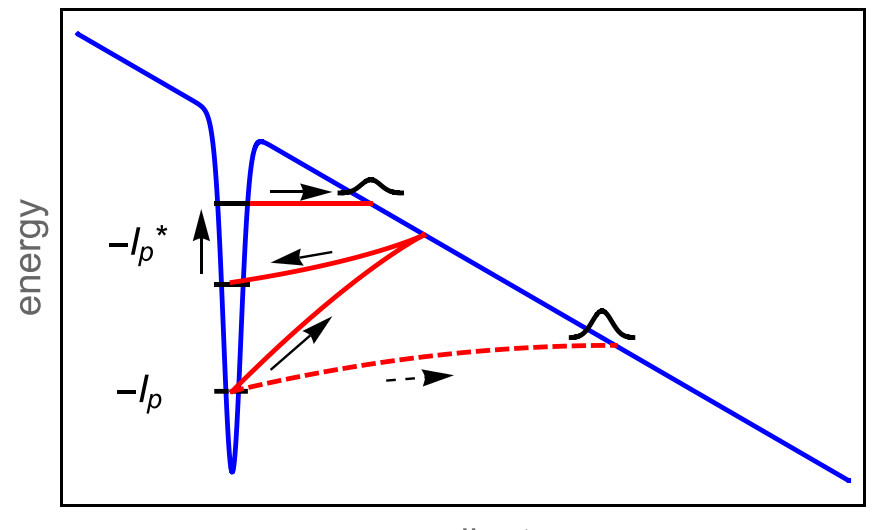

coordinate

FIG. 1. The scheme of the resonantly enhanced nonadiabatic tunneling (Freeman resonance): the sub-barrier recollision (described by a quantum trajectory propagating from the bound state up to the barrier surface, reflected, and tunneled back to the core) may yield recombination to the Stark-shifted excited state of the atom, which is followed by further ionization. The path evolution is shown by arrows. The electron energy in the excited state is up-lifted due to the laser dressing during dwelling in the excited state before further ionization. The dashed line shows the path of the direct nonadiabatic ionization.

important to have an analytical theory and, therefore, we illustrate the scheme in a simple and transparent one-dimensional model. We expect the picture to hold also in three dimensions, because the under-the-barrier recollision is virtually one dimensional along the parabolic coordinate even in the full three-dimensional consideration. The ionization dynamics of an atom in a strong laser field is described by the Hamiltonian

$$
\begin{aligned}
H & =H^{(a)}+H^{(f)}, \\
H^{(a)} & =\hat{p}^{2} / 2+V(x), \quad H^{(f)}=x E(t),
\end{aligned}
$$

where $H^{(a)}$ is the atomic Hamiltonian, $H^{(f)}$ is the laserelectron interaction Hamiltonian, $\hat{p}$ is the momentum operator, $V(x)$ is the potential of the atomic core, and $E(t)$ is the laser electric field.

The theoretical treatment is based on SFA. We begin with the exact ionization amplitude $m(p)$ for the photoelectron with final momentum $p$ :

$$
m(p)=-i \int_{t_{i}}^{t_{f}} d t\left\langle\psi_{p}^{(a)}\left(t_{f}\right)\left|U\left(t_{f}, t\right) H^{(f)}(t)\right| \psi_{0}^{(a)}(t)\right\rangle,
$$

where $U\left(t_{f}, t\right)$ is the exact time-evolution operator (TEO), $\psi_{p}^{(a)}(x, t)$ is the electron continuum eigenstate in the atomic potential $V(x)$, and $\psi_{0}^{(a)}(x, t)=\psi_{0}^{(a)}(x) \exp \left(i I_{p} t\right)$ is the wave function of the atomic ground state, with the ionization potential $I_{p}$ and with $\kappa=\sqrt{2 I_{p}}$ the atomic momentum. The linearly polarized laser pulse is described by the vector potential $A(t)=\left(E_{0} / \omega\right) f(t) \sin (\omega t)$, with the field amplitude $E_{0}$, the frequency $\omega, E(t)=-\frac{\partial A}{\partial t}$, and the slowly varying pulse envelope $f(t)$, which is switched on and off at $t_{i}$ and $t_{f}$, respectively.

We describe the strong-field ionization via resonant excitation during nonadiabatic tunneling in the third-order SFA. This pathway includes an under-the-barrier recollision due to which a transition to the excited state happens, from which the electron is readily ionized via tunneling or an over-the-barrier passage. To model the described pathway, let us begin with the exact Dyson equation related to the dynamics with the Hamiltonian of Eq. (2), in the third-order iteration:

$$
\begin{aligned}
U\left(t_{f}, t_{i}\right)= & U^{(a)}\left(t_{f}, t_{i}\right)-i \int_{t_{i}}^{t_{f}} d t U^{(f)}\left(t_{f}, t\right) H^{(f)}(t) U^{(a)}\left(t, t_{i}\right) \\
& -\int_{t_{i}}^{t_{f}} d t \int_{t^{\prime}}^{t_{f}} d t^{\prime} U^{(f)}\left(t_{f}, t^{\prime}\right) V U^{(f)}\left(t^{\prime}, t\right) H^{(f)}(t) \\
& \times U^{(a)}\left(t, t_{i}\right)+i \int_{t_{i}}^{t_{f}} d t \int_{t}^{t_{f}} d t^{\prime} \int_{t^{\prime}}^{t_{f}} d t^{\prime \prime} U^{(f)}\left(t_{f}, t^{\prime \prime}\right) \\
& \times V U\left(t^{\prime \prime}, t^{\prime}\right) V U^{(f)}\left(t^{\prime}, t\right) H^{(f)}(t) U^{(a)}\left(t, t_{i}\right),
\end{aligned}
$$

with the atomic TEO $U^{(a)}$ and the Volkov TEO $U^{(f)}$.

In Eq. (3) we need to approximate the exact TEO $U$, which is designed to describe the bound-bound transition via the sub-barrier recollision. For this reason we represent $U$ symbolically as follows:

$$
U\left(t^{\prime}, t^{\prime \prime}\right)=\sum_{n}\left|\psi_{n}\left(t^{\prime}\right)\right\rangle\left\langle\psi_{n}\left(t^{\prime \prime}\right)\left|+\int d p\right| \psi_{p}\left(t^{\prime}\right)\right\rangle\left\langle\psi_{p}\left(t^{\prime \prime}\right)\right|,
$$

with the sum running over the exact basis sets $\left|\psi_{n}(t)\right\rangle$ and $\left|\psi_{p}(t)\right\rangle$, representing the exact solutions of the Schrödinger equation in the laser and the atomic potential fields for bound and continuum states, respectively. As $U$ recounts the dynamics through the laser-dressed excited state, we extend the sum in Eq. (4) only over the bound states. Taking into account that during the resonance only one excited state $\left|\psi_{n^{*}}(t)\right\rangle$ is important in the sum Eq. (4), the one which has an energy that fits to the energy of the recolliding electron, we approximate the following:

$$
U\left(t^{\prime}, t^{\prime \prime}\right) \approx\left|\psi_{n^{*}}\left(t^{\prime}\right)\right\rangle\left\langle\psi_{n^{*}}\left(t^{\prime \prime}\right)\right| .
$$

Using Eqs. (3) and (5), we derive the third-order SFA amplitude:

$$
\begin{aligned}
m_{3}(p)= & i \int_{t_{i}}^{t_{f}} d t \int_{t}^{t_{f}} d t^{\prime} \int_{t^{\prime}}^{t_{f}} d t^{\prime \prime} \int d q \int\left\langle\psi_{p}^{(f)}\left(t^{\prime \prime}\right)|V| \psi_{n^{*}}\left(t^{\prime \prime}\right)\right\rangle \\
& \times\left\langle\psi_{n^{*}}\left(t^{\prime}\right)|V| \psi_{q}^{(f)}\left(t^{\prime}\right)\right)\left\langle\psi_{q}^{(f)}(t)|x E(t)| \psi_{0}^{(a)}(t)\right\rangle, \quad(6)
\end{aligned}
$$

where it was taken into account that in SFA the final state is free of the atomic potential $\psi_{p}^{(a)}\left(x, t_{f}\right) \approx \exp (i p x) / \sqrt{2 \pi}$. Further, we neglect the direct bound-bound transitions during the laser dressing of the bound state, assuming that the laserdressed bound state emerges from the corresponding bare bound state due to the action of the Volkov propagator:

$$
\left|\psi_{n^{*}}(t)\right\rangle \approx U^{(f)}\left(t, t_{i}\right)\left|\psi_{n^{*}}^{(a)}\left(t_{i}\right)\right\rangle,
$$

where $\left|\psi_{n^{*}}^{(a)}(t)\right\rangle$ is the corresponding bare atomic eigenstate. With Eq. (7) and the Volkov TEO $U^{(f)}\left(t^{\prime}, t^{\prime \prime}\right)=$ $\int d w\left|\psi_{w}^{(f)}\left(t^{\prime}\right)\right\rangle\left\langle\psi_{w}^{(f)}\left(t^{\prime \prime}\right)\right|$, the amplitude reads

$$
\begin{gathered}
m(p)=m_{1}(p)+m_{3}(p), \\
m_{1}(p)=-i \int_{t_{i}}^{t_{f}} d t\left\langle\psi_{p}^{(f)}(t)\left|H^{(f)}(t)\right| \psi_{0}^{(a)}(t)\right\rangle,
\end{gathered}
$$




$$
\begin{aligned}
m_{3}(p)= & i \int_{t_{i}}^{t_{f}} d t \int_{t}^{t_{f}} d t^{\prime} \int_{t^{\prime}}^{t_{f}} d t^{\prime \prime} \int d q \int d v \int d w \\
& \times\left\langle\psi_{p}^{(f)}\left(t^{\prime \prime}\right)|V| \psi_{v}^{(f)}\left(t^{\prime \prime}\right)\right\rangle\left\langle\psi_{v}^{(f)}\left(t_{i}\right) \mid \psi_{n^{*}}^{(a)}\left(t_{i}\right)\right\rangle \\
& \times\left\langle\psi_{n^{*}}^{(a)}\left(t_{f}\right) \mid \psi_{w}^{(f)}\left(t_{f}\right)\right\rangle\left\langle\psi_{w}^{(f)}\left(t^{\prime}\right)|V| \psi_{q}^{(f)}\left(t^{\prime}\right)\right\rangle \\
& \times\left\langle\psi_{q}^{(f)}(t)|x E(t)| \psi_{0}^{(a)}(t)\right\rangle,
\end{aligned}
$$

with the direct ionization amplitude $m_{1}(p)$ and the ionization amplitude with a Freeman resonance $m_{3}(p)$. To simplify the calculation of the third-order amplitude, we model the atom by a one-dimensional short-range potential, with $\langle p|V| q\rangle=$ $-\kappa /(2 \pi)$ and $\langle p|V| \phi\rangle=-\kappa^{3 / 2} / \sqrt{2 \pi}$, and use the wave function of the ground and excited states in the form $\psi_{0}^{(a)}(x)=$ $\sqrt{k} \exp (-k|x|)$, with $k=\kappa=\sqrt{2 I_{p}}$ and $k=\kappa^{*}=\sqrt{2 I_{p}^{*}}$, respectively, where $I_{p}^{*}$ is the ionization potential of the excited state.

Dressing of the bound states emerges in Eq. (10) due to transitions to intermediate Volkov states given by the integrations over the momenta $v$ and $w$. The mathematical structure of the dressing of the excited state consists of two integrals of the form

$$
\begin{aligned}
& \int d x \int d p \exp \left\{-i \int_{t_{i}}^{t} d s[p+A(s)]^{2} / 2-i p x-\kappa^{*}|x|\right\} \\
& =2 \pi \exp \left\{-i \int_{t_{i}}^{t} d s\left[i \kappa^{*}+(-1)^{k} A(s)\right]^{2} / 2\right\}
\end{aligned}
$$

which were solved with the two-dimensional SPA. Here, the factor $(-1)^{k}$ corresponds to the $k$ th half cycle and arises from the derivative of $|\alpha(t)|$, with the excursion coordinate $\alpha(t)=\int_{t_{i}}^{t} A(s) d s$. The integration over the momenta $v$ and $w$ results in dressing of the excited state with the vector potential of the laser field via $i \kappa^{*} \rightarrow i \kappa^{*}+A(s)$, effectively shifting the excited-state energy from $-I_{p}^{*}$ to $-I_{p}^{*}+U_{p}$, with the laser ponderomotive potential $U_{p}=E_{0}^{2} / 4 \omega^{2}$. The remaining integrals in $m(p)$ over the times $t, t^{\prime}, t^{\prime \prime}$, and $q$ are calculated by SPA.

We gain a physical insight of the excitation process from the saddle-point conditions. The saddle points for these four variables $t, t^{\prime}, t^{\prime \prime}$, and $q$ are determined from the following equations:

$$
\begin{gathered}
{\left[q\left(t^{\prime}, t\right)+A(t)\right]^{2} / 2=-\kappa^{2} / 2,} \\
q\left(t^{\prime}, t\right)=-\frac{\alpha\left(t^{\prime}\right)-\alpha(t)}{t^{\prime}-t}, \\
{\left[i \kappa^{*}+(-1)^{j} A\left(t^{\prime}\right)\right]^{2} / 2=\left[q\left(t^{\prime}, t\right)+(-1)^{j} A\left(t^{\prime}\right)\right]^{2} / 2,} \\
{\left[p+(-1)^{i} A\left(t^{\prime \prime}\right)\right]^{2} / 2=\left[i \kappa^{*}+(-1)^{i} A\left(t^{\prime \prime}\right)\right]^{2} / 2,}
\end{gathered}
$$

with the factors $(-1)^{i}$ and $(-1)^{j}$ corresponding to the processes in the $i$ th and $j$ th half cycles. Equations (12)-(15) describe the electron dynamics during the Freeman resonance. The ionization path (see Fig. 2) begins at a time moment $t$ from the ground state, Eq. (12). The electron leaves the ground state, tunneling through the barrier. Reaching the surface of the barrier (tunnel exit) the electron backscatters and tunnels back to the core. The electron revisits the atomic core at $t^{\prime}$, when the intermediate momentum $q\left(t^{\prime}, t\right)$ fulfills Eq. (13),
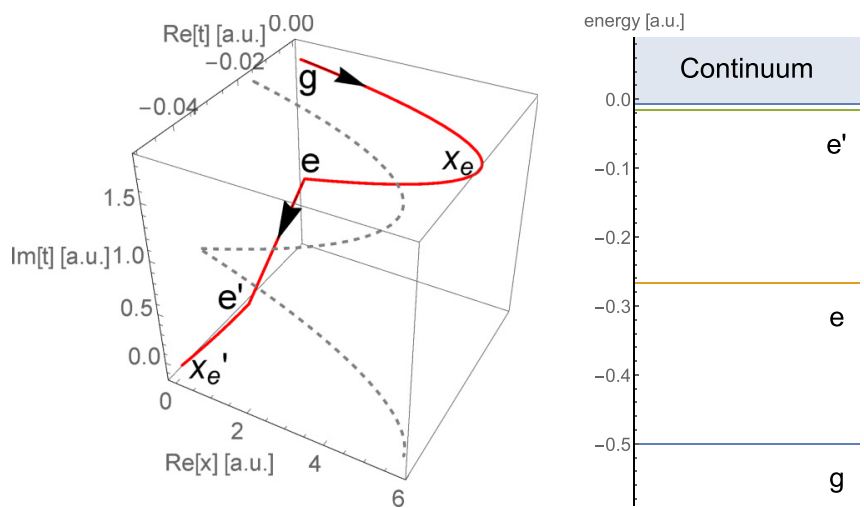

FIG. 2. The ionization paths and the coordinate $x(t)$ vs time $t=$ $\operatorname{Re}[t]+i \operatorname{Im}[t]:$ red, the sub-barrier path for the Freeman resonance begins at $t$ from the ground state $(g)$ [Eq. (12)]. The electron leaves the ground state tunneling through the barrier. Reaching the surface of the barrier $x_{e}$, the electron backscatters and tunnels back to the core, revisiting the atomic core at $t^{\prime}$ [the intermediate momentum $q\left(t^{\prime}, t\right)$ fulfills Eq. (13)], and recombines into the dressed excited state $(e)$ [see Eq. (14)]. The electron gains energy during dwelling in the excited state $\left(e^{\prime}\right)$, and finally the electron is ionized from the dressed excited state at time $t^{\prime \prime}$ [see Eq. (15)], reaching the tunnel exit again $\left(x_{e}^{\prime}\right)$. The time during the recolliding sub-barrier path $g \rightarrow$ $x_{e} \rightarrow e \rightarrow e^{\prime} \rightarrow x_{e}^{\prime}$ evolves along the imaginary axis; the direction of time is shown by arrows; the dashed line shows the ionization path with the under-the-barrier recollision: the ionization path begins from the ground state; the electron tunnels through the barrier, backscatters from the surface of the barrier, tunnels back to the core, backscatters off the core, and finally tunnels again to the tunnel exit and ionizes. In the considered nonadiabatic tunneling regime the electron gains energy during tunneling (see the right panel). The parameters are $\kappa^{*}=0.23$ a.u., $E_{0}=0.04$ a.u., and $\omega=0.05$ a.u..

which may lead to the electron recombination into the dressed excited state [see Eq. (14)]. In the considered nonadiabatic tunneling regime the electron gains energy during tunneling, which allows for the transition to the dressed excited state. Finally, the electron is ionized from the dressed excited state at time $t^{\prime \prime}$, described by Eq. (15). The time during this recolliding sub-barrier trajectory evolves along the imaginary axis.

\section{RESULTS}

\section{A. The half-cycle contribution to the yield}

First, we examine the excitation of a Rydberg state via sub-barrier recollision. Let us analyze the contribution to the excitation yield $Y_{\mathrm{ex}}$ during a half cycle of the laser field. We define $Y_{\mathrm{ex}} \equiv \int\left|m_{\mathrm{ex}}(p)\right|^{2} d p$, with the excitation amplitude due to sub-barrier rescattering:

$$
\begin{aligned}
m_{\mathrm{ex}}= & i \int_{t_{i}}^{t_{f}} d t \int_{t}^{t_{f}} d t^{\prime} \int d q \int d w\left\langle\psi_{n^{*}}\left(t_{f}\right) \mid \psi_{w}^{(f)}\left(t_{f}\right)\right\rangle \\
& \times\left\langle\psi_{w}^{(f)}\left(t^{\prime}\right)|V| \psi_{q}^{(f)}\left(t^{\prime}\right)\right\rangle\left\langle\psi_{q}^{(f)}(t)\left|H^{(f)}(t)\right| \psi_{0}^{(a)}(t)\right\rangle,
\end{aligned}
$$

which is derived from the second-order term in Eq. (3), using the excited bound state with a time evolution as in Eq. (7) as final state and the atomic ground state as initial state. The ratio of the resonant excitation to the direct ionization yield, $Y_{\mathrm{ex}} / Y_{1}$, 

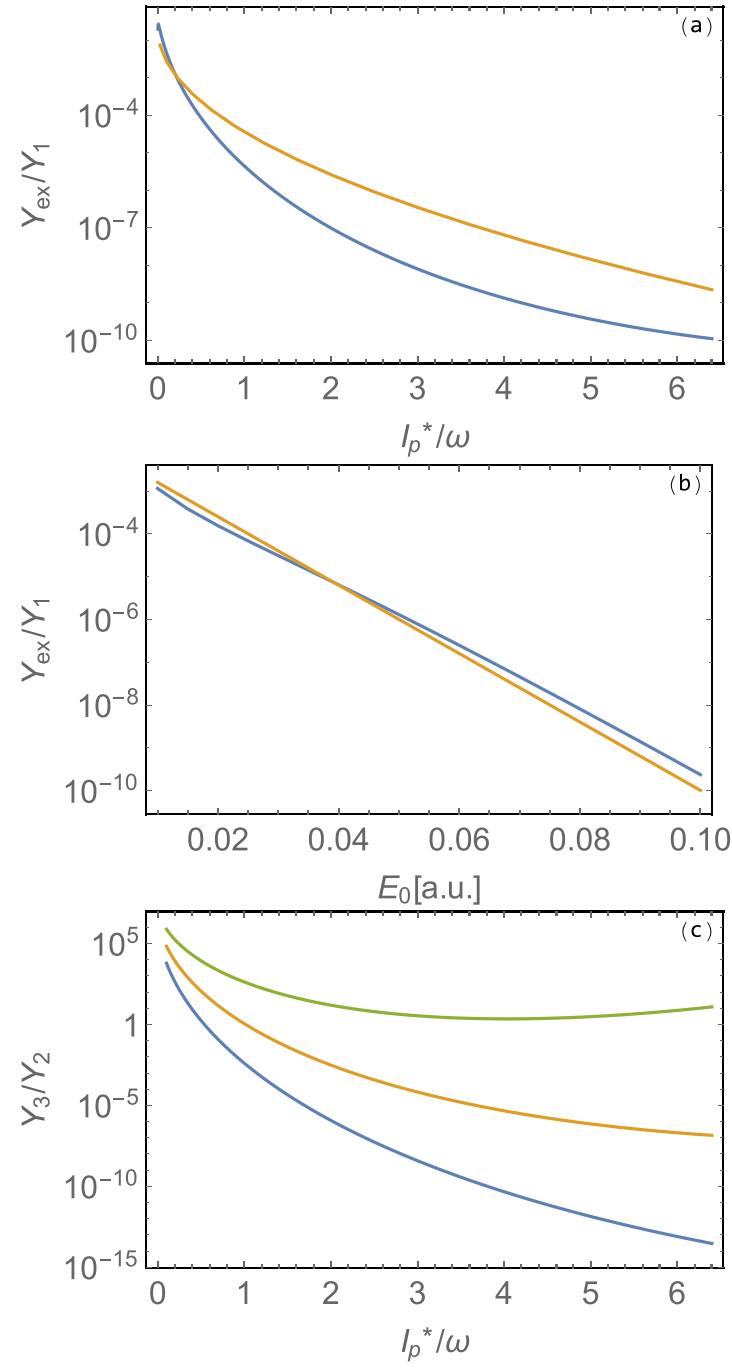

FIG. 3. Ratio of the excitation yield to that of the direct ionization $Y_{\mathrm{ex}} / Y_{1}$ (blue) from a single half cycle: (a) vs $\kappa^{*}$, for $E_{0}=0.025$ a.u. and (b) vs the laser field amplitude $E_{0}$ for $\kappa^{*}=0.23$ a.u.; $\omega=0.05$ a.u., $\kappa=1$ a.u., $\gamma=2$; orange, the scaling $\sim \exp \left(-2 \kappa^{*} \alpha\right)$. (c) Ratio $Y_{3} / Y_{2}$ of the ionization yields due to the sub-barrier recollision with $\left(Y_{3}\right)$ and without $\left(Y_{2}\right)$ excited state, respectively, for $E_{0}=0.035$ a.u. (blue), $E_{0}=0.025$ a.u. (orange), and $E_{0}=0.015$ a.u. (green).

is shown in Figs. 3(a) and 3(b), where $Y_{1}=\int\left|m_{1}(p)\right|^{2} d p$. We see that the excitation during a half cycle is quite small. The excitation probability is significantly damped at large $I_{p}^{*}$ and at large fields, which can be explained as follows. The process takes place at the laser field maximum, when the spatial distribution of the dressed excited state is concentrated at the distance $\alpha \sim E_{0} / \omega^{2}$ away from the core, with the width $\approx 1 / \kappa^{*}$; meanwhile, for recombination the recolliding electron arrives at the core, because momentum transfer from the core is needed for recombination. As a result the recombination into the excited state is suppressed by a factor $\exp \left(-2 \kappa^{*} \alpha\right)$ [see Figs. 3(a) and 3(b)].

The tunneling via the sub-barrier recolliding path is also possible without excitation to the Rydberg state, however, with a relatively small probability [23]. How the availability of the intermediate excited state changes the probability of the sub-barrier path is demonstrated in Fig. 3(c), where the ratio $Y_{3} / Y_{2}$ of the ionization yield during a half-cycle period due to the sub-barrier recollision with $\left(Y_{3}=\int\left|m_{3}\right|^{2} d p\right)$ and without $\left(Y_{2}=\int\left|m_{2}\right|^{2} d p\right)$ excited state is shown. Here, the sub-barrier recollision is described in the second-order SFA by the matrix element

$$
\begin{aligned}
m_{2}= & -\int_{t_{i}}^{t_{f}} d t \int_{t}^{t_{f}} d t^{\prime} \int d q\left\langle\psi_{p}^{(f)}\left(t^{\prime}\right)|V| \psi_{q}^{(f)}\left(t^{\prime}\right)\right\rangle \\
& \times\left\langle\psi_{q}^{(f)}(t)\left|H^{(f)}(t)\right| \psi_{0}^{(a)}(t)\right\rangle .
\end{aligned}
$$

For the description of the sub-barrier recolliding path, the time integrations Eq. (17) should be carried out by SPA including the trajectory running along the imaginary time axis [23]. Then the ionization path begins from the ground state, and the electron tunnels through the barrier, backscatters from the surface of the barrier, tunnels back to the core, backscatters off the core, and finally tunnels again to the tunnel exit and ionizes (see dashed line in Fig. 2).

During the half cycle there is no resonance enhancement in the excitation, which is created only due to multicycle interference. Nevertheless, we see from Fig. 3(c) that even in that case the intermediate bound state increases the ionization probability several times at small energies of the excited state $I_{p}^{*}$ and weak fields. There are two reasons for this enhancement. First, the electron gains energy during the dwelling in the excited state, which increases further the ionization probability due to a shorter tunneling path. Second, the electron wave-packet longitudinal spreading is suppressed during the dwelling time in the excited state. The spreading factor is dominating, and it is larger for larger Keldysh parameters, therefore the enhancement due to absence of spreading is also larger for larger $\gamma$. Figure 3(c) also shows that $Y_{3} / Y_{2} \ll 1$ at large fields and large ionization energies of the excited state. This is because the recombination into the excited state is suppressed by the factor $\approx \exp \left(-2 \kappa^{*} \alpha\right)$, as noted above and in Figs. 3(a) and 3 (b). While the enhancement of the ionization yield due to the transient excitation is not large in the half-cycle contribution, it is significantly boosted due to multicycle interference, as discussed below.

\section{B. Multicycle interference}

A conspicuous resonance effect emerges in a long laser pulse when interference of contributions in the ionization amplitudes from different laser cycles is included. The structure of the $m_{3}$ amplitude is a product of an amplitude of direct ionization and a recombination amplitude into the dressed excited state. We take the following multicycle effects into account: most importantly, the intercycle interference of the excitation amplitudes, which induces the Freeman resonances; second, the usual intercycle interference of the ionization amplitudes, which gives the photon structure in a photoelectron spectrum. We allow also for multiple cycles between excitation and ionization from the excited state.

The structure of the $m_{3}$ amplitude after SPA is a product of an amplitude of direct ionization and a recombination amplitude into the dressed excited state:

$$
m_{3}(p)=\sum_{t_{s}^{\prime \prime}>t_{s}^{\prime}>t_{s}} m_{1}^{*}\left(t_{s}^{\prime \prime}\right) m_{\mathrm{ex}}\left(t_{s}, t_{s}^{\prime}\right) .
$$


The three-dimensional saddle points are decoupled and the direct ionization can happen in a different subsequent half cycle than the rescattering recombination. Taking into account the single physical saddle points in $t$ and $t^{\prime}$ SPA, we can have a simplified representation via the direct ionization and excitation amplitudes $m_{1, i}^{*}$ and $m_{\mathrm{ex}, j}$ in the corresponding half cycles $i$ and $j$, respectively:

$$
m_{3}(p)=\sum_{i} m_{1, i}^{*} \sum_{j \leqslant i} m_{\mathrm{ex}, j}
$$

where the time-ordering ionization after recombination is ensured by $j \leqslant i$. The phase of the excitation amplitude has the form

$$
\begin{aligned}
\Phi_{j}= & (-1)^{j} \kappa^{*}\left[\alpha\left(t^{\prime}\right)-\alpha\left(t_{i}\right)\right]-\left[\frac{\kappa^{* 2}}{2} t^{\prime \prime}-\beta\left(t^{\prime \prime}\right)+\beta\left(t_{i}\right)\right] \\
& -\int_{t^{\prime \prime}}^{t^{\prime}} d s \frac{\left[q\left(t^{\prime}, t^{\prime \prime}\right)+A(s)\right]^{2}}{2}+\frac{\kappa^{2}}{2} t^{\prime \prime} \\
& +\frac{1-\mathcal{P}_{e}}{2} i \log [\alpha(t)],
\end{aligned}
$$

where $j$ is the half-cycle number, $\dot{\beta}(t)=A^{2}(t) / 2$, and $\mathcal{P}_{e}$ is the parity of the excited state. Two consecutive half cycles with $t^{\prime} \rightarrow t^{\prime}+\pi / \omega$ and $t^{\prime \prime} \rightarrow t^{\prime \prime}+\pi / \omega$ have the phase difference $\Delta \Phi=\pi\left(U_{p}+I_{p}^{*}-I_{p}\right) / \omega+\pi\left(1-\mathcal{P}_{e}\right) / 2$. The interference of excitation amplitudes in the second sum of Eq. (19) is constructive, and the yield is enhanced, if the resonance condition is fulfilled:

$$
U_{p}+I_{p}^{*}-I_{p}=\ell \omega,
$$

with an integer $\ell$; even $\ell$ corresponds to the case of the same parity of the ground and the excited states, and odd $\ell$ corresponds to the opposite parities. For the given excited state, the resonance peaks with respect to the state energy (or the laser intensity) have $2 \omega$ separation (see Fig. 4). This is due to constructive interference of the excitation amplitudes originating from each half cycle. Comparison of the multicycle yield in Fig. 4 with that of a single half-cycle one of Fig. 3(a) shows that the yield $Y_{\mathrm{ex}} / Y_{1}$ scales roughly quadratically with the number of laser cycles. This is because the resonance comes from the coherent contributions of half-cycle terms in the sum $\sum_{j} m_{\mathrm{ex}, j}$ in Eq. (19). Our model includes only one (lowest) excited state; more resonances are possible when higher excited states are considered.

The excitation yield dependence on the laser intensity is illustrated in Fig. 4. Again we see the enhancement of the excitation yield at the resonance conditions $\left(U_{p}-I_{p}^{*}+I_{p}\right)=$ $2 \ell \omega$, with an integer $\ell$. There is no significant singular behavior of the yield at the channel closing condition $I_{p}+U_{p}=n \omega$ (dashed grid lines), because the latter effect [24-26] is described by $m_{2}$, while the excitation yield is based on $m_{3}$.

\section{Photoelectron spectra}

We analyze the signature of the resonant transient excitation during strong-field ionization in the photoelectron spectra. Photoelectron energy distribution in a ten-cycle laser pulse is shown in Fig. 5 in the case of the resonant excitation. We see that our model of excitations via sub-barrier recollision is able to describe the typical photoelectron spectra at
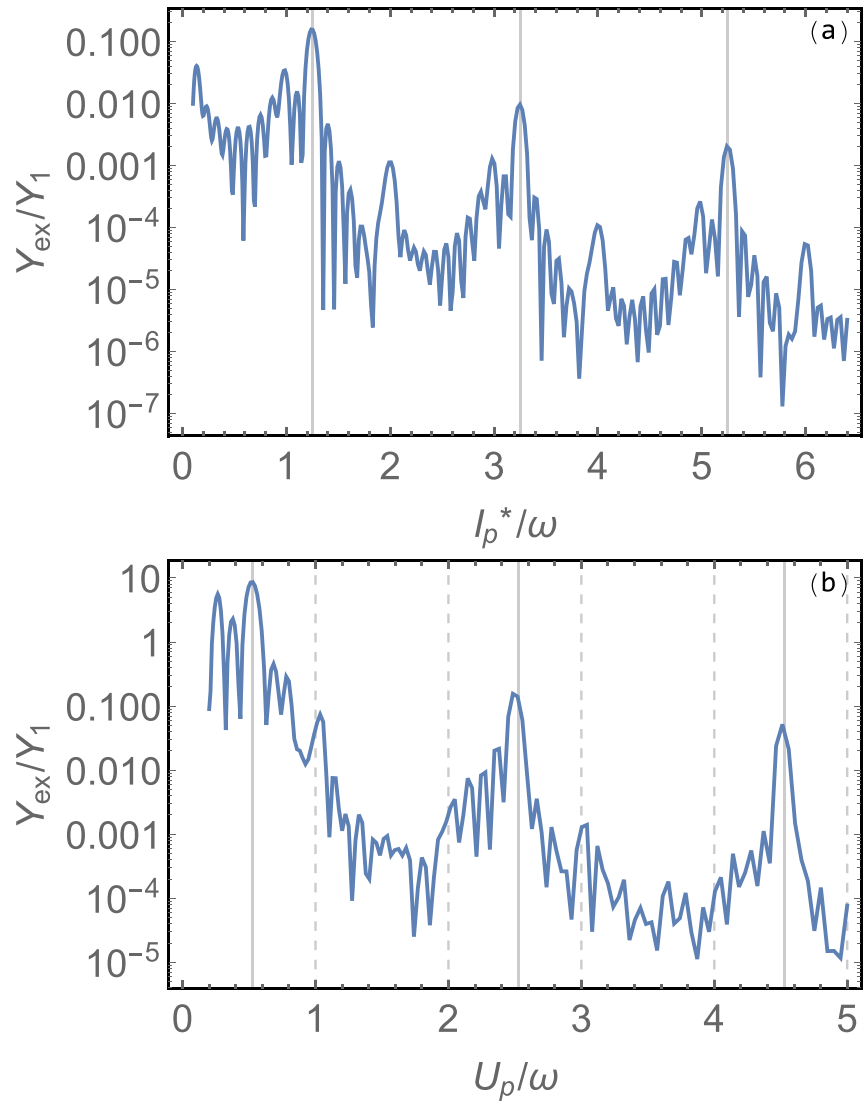

FIG. 4. Ratio of the excitation yield to that of the direct ionization $Y_{\mathrm{ex}} / Y_{1}$ from ten cycles: (a) vs $I_{p}^{*} / \omega$ and (b) vs $U_{p} / \omega$, for $E_{0}=$ 0.025 a.u, $\omega=0.05$ a.u. $(\gamma=2$ for the ground state $\kappa=1)$. The resonance conditions $\left(U_{p}-I_{p}^{*}+I_{p}\right)=2 \ell \omega$, with an integer $\ell$, for the given excited state $\left(\mathscr{P}_{e}=1\right)$ are indicated by vertical solid lines. The channel-closing resonance condition $I_{p}+U_{p}=n \omega$ is indicated by dashed vertical lines.

Freeman resonances. A double peak structure arises in the spectrum (see Fig. 5). One peak in series corresponds to the direct ionization from the ground state with the energy conservation $n \omega=p^{2} / 2+U_{p}+I_{p}$. The second peak in series is due to the Freeman resonance. It corresponds to the multiphoton transition from the excited state with the energy $-I_{p}^{*}+U_{p}$ to the continuum with the energy $p^{2} / 2+U_{p}$, with the energy conservation $n \omega=p^{2} / 2+I_{p}^{*}$ (see grid lines in Fig. 5).

\section{CONCLUSION}

We have proposed an intuitive model for Freeman resonances in the nonadiabatic tunneling ionization. Using specific quantum orbits provided by strong-field approximation theory, we show a concrete pathway leading to the transient population of intermediate excited states during strong-field ionization. What is interesting is the pathway along which the electron gains energy necessary for the transfer to the excited state: It mostly travels under the barrier, reflects from the outer surface of the barrier, propagates back to the core, and recombines to the excited bound state. All this sub-barrier recollision takes place during imaginary time within a single half cycle, visualizing the electron vertical 


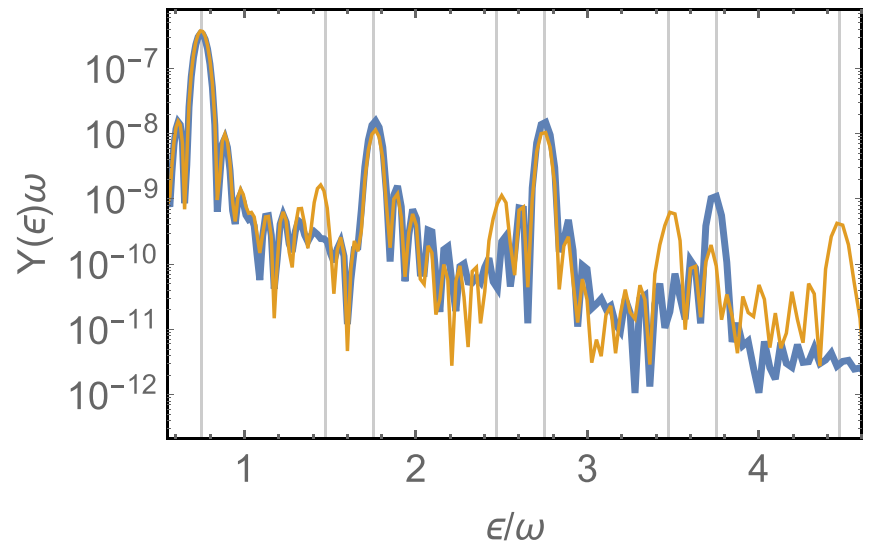

FIG. 5. Photoelectron energy spectrum in a ten-cycle laser pulse in a logarithmic scale: blue, direct ionization via $m_{1}$; orange, ionization at a Freeman resonance via $m_{1}+m_{3} ; E_{0}=0.025$ a.u., $\omega=$ 0.05 a.u., $\kappa^{*}=0.23$ a.u., $\kappa=1$ a.u., $\gamma=2$. Grid lines indicate the ionization from the ground state $n-\left(I_{p}+U_{p}\right) / \omega$ and from the excited state $n-I_{p}^{*} / \omega$.

transition in the strongly driven atom [18]. We found that the available excited bound state can increase the probability of the sub-barrier recolliding pathway, even during the single half-cycle contribution. The latter is mostly due to the suppressed spreading of the electron wave packet, during the dwelling time in the excited state. Although the transition probability during a half cycle is small, it is resonantly enhanced due to constructive interference of contributions to the excitation amplitude emerging from different laser half cycles, proportional to the square of the number of half cycles. As each half cycle gives an interfering contribution, the resonance conditions of different orders for a certain excited state in this model are separated by twice the photon energy. The described sub-barrier pathway of the Freeman resonance is relevant in the nonadiabatic tunneling regime, when the electron gains energy during tunneling, enabling transition to the laser dressed excited state.

As an outlook beyond the scope of this paper, let us note a possible application of the presented model of the Freeman resonances via sub-barrier recollision on the strong-field electron-positron pair production problem in ultrastrong laser fields. When pairs are produced during the impinging of the laser beam on a nucleus (ion, or other atomic system), then the produced electron from vacuum due to multiphoton process can be captured into the bound state in the Coulomb potential. This bound-free channel of pair production has been thoroughly investigated in Refs. [27-31]. However, rather than real bound-free pair production, the state of a bound-free pair can emerge virtually as a transition state, which finally may end up with a free electron and positron state. This pathway resembles conceptually the Freeman resonance discussed in this paper. The present model of under-the-barrier recollision can be extended for the solution of the Dirac equation describing excitation of the Dirac sea electron into a positive energy state, with transient capture into the bound state.
[1] R. R. Freeman, P. H. Bucksbaum, H. Milchberg, S. Darack, D. Schumacher, and M. E. Geusic, Above-Threshold Ionization with Subpicosecond Laser Pulses, Phys. Rev. Lett. 59, 1092 (1987).

[2] P. Agostini, P. Breger, A. L'Huillier, H. G. Muller, G. Petite, A. Antonetti, and A. Migus, Giant Stark Shifts in Multiphoton Ionization, Phys. Rev. Lett. 63, 2208 (1989).

[3] R. R. Freeman and P. H. Bucksbaum, Investigations of abovethreshold ionization using subpicosecond laser pulses, J. Phys. B 24, 325 (1991).

[4] E. Mevel, P. Breger, R. Trainham, G. Petite, P. Agostini, A. Migus, J. P. Chambaret, and A. Antonetti, Atoms in Strong Optical Fields: Evolution from Multiphoton to Tunnel Ionization, Phys. Rev. Lett. 70, 406 (1993).

[5] M. J. Nandor, M. A. Walker, L. D. Van Woerkom, and H. G. Muller, Detailed comparison of above-thresholdionization spectra from accurate numerical integrations and high-resolution measurements, Phys. Rev. A 60, R1771(R) (1999).

[6] R. Wiehle, B. Witzel, H. Helm, and E. Cormier, Dynamics of strong-field above-threshold ionization of argon: Comparison between experiment and theory, Phys. Rev. A 67, 063405 (2003).

[7] T. Morishita, Z. Chen, S. Watanabe, and C. D. Lin, Twodimensional electron momentum spectra of argon ionized by short intense lasers: Comparison of theory with experiment, Phys. Rev. A 75, 023407 (2007).
[8] Y. Wang, J. Zhang, Z. Xu, Y.-S. Wu, J. T. Wang, and D.-S. Guo, Direct theoretical method for the determination of peak laser intensities from Freeman resonances in above-threshold ionization, Phys. Rev. A 80, 053417 (2009).

[9] R. M. Potvliege and S. Vučić, Freeman resonances in highorder above-threshold ionization, J. Phys. B 42, 055603 (2009).

[10] M. Li, P. Zhang, S. Luo, Y. Zhou, Q. Zhang, P. Lan, and P. Lu, Selective enhancement of resonant multiphoton ionization with strong laser fields, Phys. Rev. A 92, 063404 (2015).

[11] N. A. Hart, J. Strohaber, A. A. Kolomenskii, G. G. Paulus, D. Bauer, and H. A. Schuessler, Selective strong-field enhancement and suppression of ionization with short laser pulses, Phys. Rev. A 93, 063426 (2016).

[12] P. Stammer, S. Patchkovskii, and F. Morales, Evidence of ac-stark-shifted resonances in intense two-color circularly polarized laser fields, Phys. Rev. A 101, 033405 (2020).

[13] D. Chetty, R. D. Glover, B. A. deHarak, X. M. Tong, H. Xu, T. Pauly, N. Smith, K. R. Hamilton, K. Bartschat, J. P. Ziegel, N. Douguet, A. N. Luiten, P. S. Light, I. V. Litvinyuk, and R. T. Sang, Observation of dynamic stark resonances in strong-field excitation, Phys. Rev. A 101, 053402 (2020).

[14] L. V. Keldysh, Ionization in the field of a strong electromagnetic wave, Zh. Eksp. Teor. Fiz. 47, 1945 (1964) [Sov. Phys. JETP 20, 1307 (1965)].

[15] F. H. M. Faisal, Multiple absorption of laser photons by atoms, J. Phys. B 6, L89 (1973). 
[16] H. R. Reiss, Effect of an intense electromagnetic field on a weakly bound system, Phys. Rev. A 22, 1786 (1980).

[17] G. L. Yudin and M. Yu. Ivanov, Nonadiabatic tunnel ionization: Looking inside a laser cycle, Phys. Rev. A 64, 013409 (2001).

[18] M. Yu. Ivanov, M. Spanner, and O. Smirnova, Anatomy of strong field ionization, J. Mod. Opt. 52, 165 (2005).

[19] M. Klaiber, K. Z. Hatsagortsyan, and C. H. Keitel, Tunneling Dynamics in Multiphoton Ionization and Attoclock Calibration, Phys. Rev. Lett. 114, 083001 (2015).

[20] P. Salières, B. Carré, L. Le Déroff, F. Grasbon, G. G. Paulus, H. Walther, R. Kopold, W. Becker, D. B. Milošević, A. Sanpera, and M. Lewenstein, Feynman's path-integral approach for intense-laser-atom interactions, Science 292, 902 (2001).

[21] W. Becker, F. Grasbon, R. Kopold, D. B. Milosšević, G. G. Paulus, and H. Walther, Above-threshold ionization: from classical features to quantum effects, Adv. Atom. Mol. Opt. Phys. 48, 35 (2002).

[22] P. B. Corkum, Plasma Perspective on Strong Field Multiphoton Ionization, Phys. Rev. Lett. 71, 1994 (1993).

[23] M. Klaiber, K. Z. Hatsagortsyan, and C. H. Keitel, Underthe-Tunneling-Barrier Recollisions in Strong-Field Ionization, Phys. Rev. Lett. 120, 013201 (2018).

[24] G. G. Paulus, F. Grasbon, H. Walther, P. Villoresi, M. Nisoli, S. Stagira, E. Priori, and S. De Silvestri, Absolute-phase phenomena in photoionization with few-cycle laser pulses, Nature (London) 414, 182 (2001).

[25] S. V. Popruzhenko, P. A. Korneev, S. P. Goreslavski, and W. Becker, Laser-Induced Recollision Phenomena: Interference Resonances at Channel Closings, Phys. Rev. Lett. 89, 023001 (2002).

[26] J. Wassaf, V. Véniard, R. Taïeb, and A. Maquet, Strong Field Atomic Ionization: Origin of High-Energy Structures in Photoelectron Spectra, Phys. Rev. Lett. 90, 013003 (2003).

[27] C. Müller, A. B. Voitkiv, and N. Grün, Nonlinear BoundFree Pair Creation in the Strong Electromagnetic Fields of a Heavy Nucleus and an Intense X-ray Laser, Phys. Rev. Lett. 91, 223601 (2003).

[28] C. Müller, A. B. Voitkiv, and N. Grün, Few-photon electronpositron pair creation in the collision of a relativistic nucleus and an intense x-ray laser beam, Phys. Rev. A 70, 023412 (2004).

[29] C. Müller, C. Deneke, and C. H. Keitel, Muon-Pair Creation by Two X-ray Laser Photons in the Field of an Atomic Nucleus, Phys. Rev. Lett. 101, 060402 (2008).

[30] C. Deneke and C. Müller, Bound-free $e^{+} e^{-}$pair creation with a linearly polarized laser field and a nuclear field, Phys. Rev. A 78, 033431 (2008).

[31] J. Sommerfeldt, R. A. Müller, A. N. Artemyev, and A. Surzhykov, Polarization effects in bound-free pair production, Phys. Rev. A 100, 042511 (2019). 